\title{
Doppler imaging of the active star PW And
}

\author{
Sheng-hong Gu${ }^{1}$, Andrew Collier Cameron ${ }^{2}$ and Kang Min Kim ${ }^{3}$ \\ ${ }^{1}$ National Astronomical Observatories/Yunnan Observatory, Chinese Academy of Sciences, \\ P.O. Box 110, Kunming 650011, P. R. China \\ email: shenghonggu@ynao.ac.cn \\ ${ }^{2}$ SUPA, School of Physics and Astronomy, University of St Andrews, North Haugh, St \\ Andrews, Fife KY16 9SS, UK \\ ${ }^{3}$ Korea Astronomy and Space Science Institute, 61-1 Whaam-Dong Yuseong-Gu, Daejeon \\ 305-348, Korea
}

\begin{abstract}
We present spot activity of the $\mathrm{K} 2 \mathrm{~V}$ pre-main-sequence star $\mathrm{PW}$ And based on the high-resolution spectroscopic data obtained at Xinglong station and BOAO in 2005 November and December. Using the Least-Squares Deconvolution method, we have derived time series of composite profiles of PW And with high signal to noise ratio. These have been used to reconstruct its starspot pattern via the Doppler imaging method. The result shows that intermediate to highlatitude spots are the dominant features and weak low-latitude spots also appear. Comparing Doppler images from two data subsets, it can be found that almost no difference between two images exists except the small position evolution of weak low-latitude spots, which suggests that the intermediate to high-latitude spots have longer lifetimes than one month, and the low-latitude spots have shorter lifetimes.
\end{abstract}

Keywords. stars: late-type, stars: activity, stars: spots

\section{Introduction}

PW And (HD1405) is one member of nearby AB Dor moving group (Montes et al. 2001b, Zuckerman \& Song 2004). It is a young pre-main-sequence K2V dwarf with a large rotation velocity vsini $=22-24 \mathrm{~km} / \mathrm{s}$ (Lopez-Santiago et al. 2003, Strassmeier \& Rice 2006). Previous results show that PW And is an active star with starspots and chromospheric emission (Bidelman 1985, Hooten \& Hall 1990, Montes et al. 2001a, Lopez-Santiago et al. 2003, Strassmeier \& Rice 2006).

\section{Observations and data reduction}

PW And was observed at Xinglong station of National Astronomical Observatories, China (NAOC) on 2005 November 18-24 and December 17-22 using the 2.16m telescope and Coudé echelle spectrograph with a $1 \mathrm{k} \times 1 \mathrm{k}$ CCD detector (Zhao \& Li 2001), and Bohyunsan Optical Astronomy Observatory, Korea (BOAO) on 2005 December 08-11 using the $1.8 \mathrm{~m}$ telescope and BOES spectrograph with a $2 \mathrm{k} \times 4 \mathrm{k}$ CCD detector (Kim et al. 2002, Kim et al. 2007). The spectral resolution is about 36000 for Xinglong observations and about 48000 for BOAO one.

The observed data are reduced using IRAF package following standard procedure, including bias subtraction, flat fielding, background subtraction, cosmic ray removal, optimal extraction, wavelength calibration and continuum fitting. 


\section{Image reconstruction}

In order to check whether the spot pattern evolves during our observing period spanned just more than one month, we separate the all data to two data subsets to do Doppler imaging respectively. Data subset 1 is corresponding to the observations made on Nov.1824 (Xinglong) and Dec. 8-11 (BOAO), 2005, data subset 2 to the observations on Dec.8-11 (BOAO) and Dec.17-22 (Xinglong), 2005. To obtain time series of line profiles with the highest signal to noise ratios from the data for Doppler imaging purpose, we employ the Least-Squares Deconvolution (LSD) method (Donati et al. 1997) to combine the profile information from all the observed photospheric lines in each observed spectrum.

We use the single star mode of our Doppler imaging code DoTS (Collier Cameron 1997) to reconstruct the spot pattern of PW And based on the above LSD profiles. The resulting Doppler images based on two data subsets are shown in Fig. 1 where the mean spot filling vs. latitude is also displayed.

\section{Discussion and conclusions}

In Fig. 1, it can be seen that the predominant features of Doppler images of PW And in our observing season are intermediate to high-latitude spots, which are obviously different from the results derived by Strassmeier \& Rice (2006), which show no spots appeared above the latitude $40^{\circ}$.
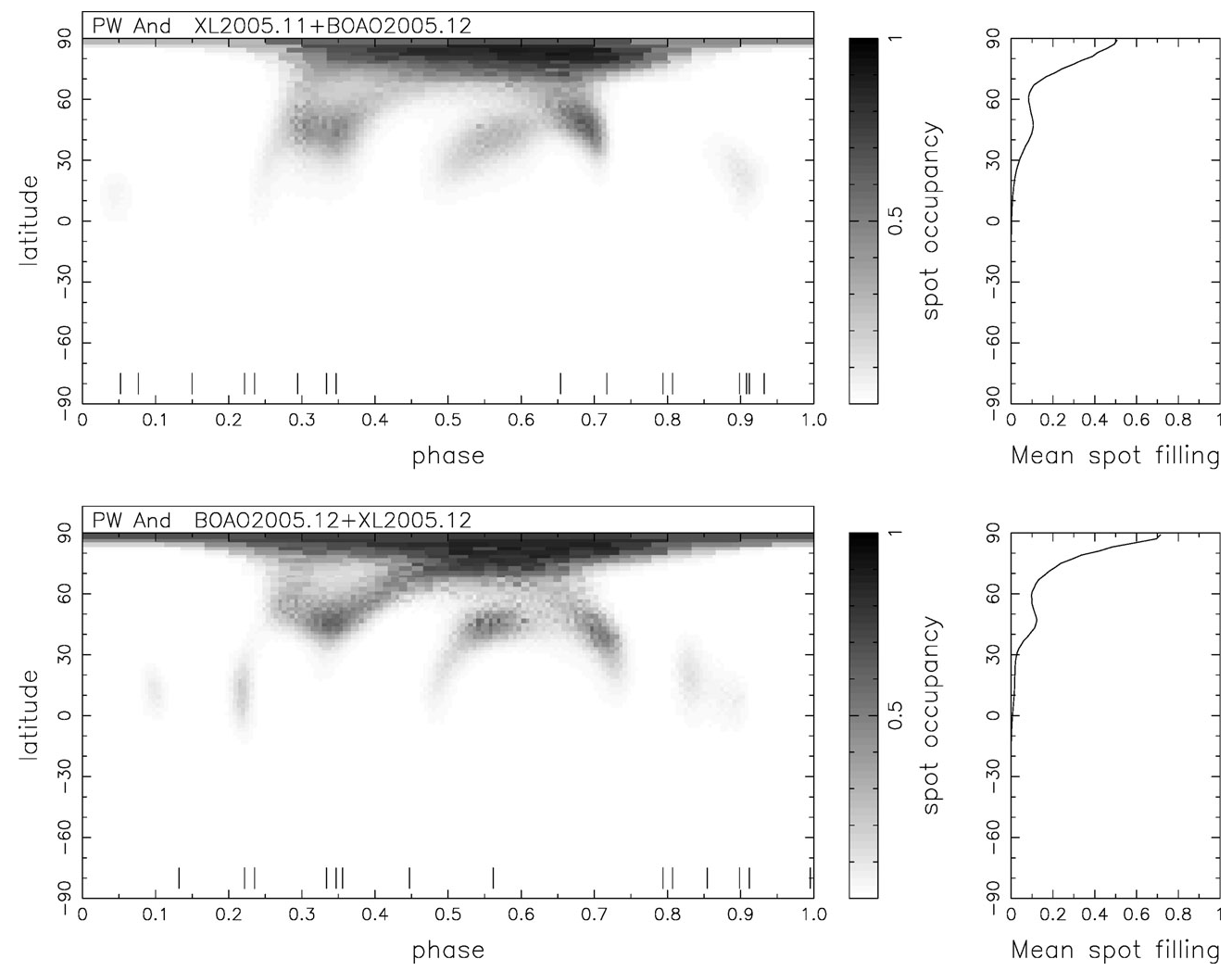

Figure 1. Doppler images of PW And for two data subsets. The upper image is for the data subset 1 and the lower one for the data subset 2 . 
From the Doppler images in Fig. 1, it is clear that two spot patterns are almost the same as each other except small low-latitude spots. This indicates that the main structure of spot pattern is stable during one month time scale. However, the low-latitude spots have some changes either in position or in intensity. Such a result implies that the lowlatitude spots seem to have shorter lifetimes than the intermediate to high-latitude spots, like most of other magnetic active stars.

Hooten \& Hall (1990) obtained four groups of photometric data, and found the second dataset spanned more than 5 months gave a large scatter when searching the period although it contains the largest number of data points, whereas the fourth dataset spanned less than one month gave a small scatter for the period obtained. This suggests that the spot pattern is stable for short duration, and changes for long time interval.

From the observations collected in Aug. 2004, Strassmeier \& Rice (2006) derived a spot pattern similar to our Sun, in which the six main cool spots concentrate below latitude $40^{\circ}$. From new observations made in Nov. and Dec. 2005, we get a obviously different spot pattern with the main spots distributed from latitude $41^{\circ}$ to $83^{\circ}$. Such a large difference suggests that the lifetime of main spot structure cannot last more than one year.

These information suggests that the spot pattern of PW And should change largely during its one activity cycle, like another active single star LQ Hya with the same spectral type $(\mathrm{K} 2 \mathrm{~V})$ and similar rotational velocity, whose Doppler imaging results show that sometimes the low-latitude spots were predominant, and the obvious polar spots appeared in other times (Donati 1999, Donati et al. 2003, kovari et al. 2004).

\section{Acknowledgments}

$\mathrm{Gu}$ and Cameron acknowledge the support of Chinese Academy of Sciences and the Royal Society through an International Joint Project award that provided travel funds and living expenses during Gu's two visits to the University of St Andrews, and Cameron's visit to Yunnan Observatory in the course of this work. This work is also supported by NSFC under grants No. 10373023 and No. 10773027.

\section{References}

Bidelman, W. P. 1985, AJ, 90, 341

Collier Cameron, A. 1997, MNRAS, 287, 556

Donati, J.-F. 1999, MNRAS, 302, 457

Donati, J.-F., Collier Cameron, A., Semel, M. et al. 2003, MNRAS, 345, 1145

Donati, J.-F., Semel, M., Carter, B. D. et al. 1997, MNRAS, 291, 658

Hooten, J. T. \& Hall, D. S. 1990, ApJS, 74, 225

Kim, K. M., Han, I., Valyavin, G. et al. 2007, PASP, 119, 1052

Kim, Kang-Min, Jang, Be-Ho, Han, Inwoo et al. 2002, JKAS, 35, 221

Kovari, Zs., Strassmeier, K. G., Granzer, T. et al. 2004, A\& $A, 417,1047$

Lopez-Santiago, J., Montes, D., Fernandez-Figueroa, M. J. et al. 2003, A\&\&A, 411, 489

Montes, D., Lopez-Santiago, J., Fernandez-Figueroa, M. J. et al. 2001a, A\& A, 379, 976

Montes, D., Lopez-Santiago, J., Galvez, M. C. et al. 2001b, MNRAS, 328, 45

Strassmeier, K. G. \& Rice, J. B. 2006, A\&A, 460, 751

Zhao, G. \& Li, H.-B. 2001, ChJAA, 1, 555

Zuckerman, B. \& Song, I. 2004, ApJ, 613, L65 